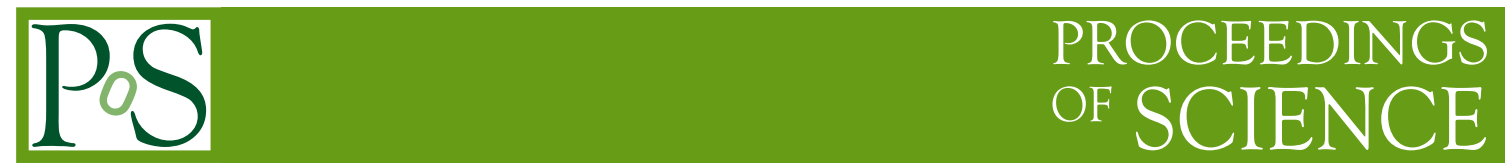

\title{
Correlators in radioastronomy
}

\author{
G Comoretto* \\ Osservatorio di Arcetri \\ E-mail: (comore earcetri.astro.it).
}

The signal received by a radiotelescope can be analyzed in several ways. A review of the most common continuum, spectroscopic, and interferometric backends is presented. These instruments are currently being implemented using completely digital techniques.

The First MCCT-SKADS Training School

September 23-29 2007

Medicina Bologna, Italy

${ }^{*}$ Speaker. 


\section{Radioastronomic backends}

The radio signal (electric field) $E(t)$ collected by the telescope and processed by the receiver and IF amplifier is amplified by a factor around $10^{8}$, translated in frequency in order to be more easily analyzed, and limited in band to a final bandwidth of a few GHz. The resulting signal is then processed in the so called back-end.

In general the astronomer is interested in measuring the incoming spectral density $S(v)$ as a function of time, frequency, direction, polarization, etc., or a combination of these variables. Accordingly, the signal is analyzed by different instruments (different back-ends), or using different modes of the same instruments.

In many cases the IF bandwidth is too large to be processed at once, This may be due to instrumental limitations, because the back-end can operate only on a smaller portion of the available bandwidth. For example correlators cannot usually analyze a band larger than a few $100 \mathrm{MHz}$. In other cases the spectral range is limited by scientific needs, for example in spectroscopic observations of narrow lines. In other cases a limited spectral resolution is needed, for example Faraday rotation may decorrelate a polarimetric measurement of the signal if the bandwidth exceeds a few $10 \mathrm{MHz}$.

In all these cases the receiver output is further processed in a smaller heterodyne receiver that extracts a portion of the total available bandwidth, and converts it to a lower frequency. This receiver is often called a Baseband converter, or BBC, and usually the receiver system includes several (4-16) BBCs, each followed by a separate back-end. In this way, a wider effective spectral range can be synthesized.

\section{Continuum backends}

\subsection{Total power measurements}

The simplest observable is the total integrated flux (total power) over the receiver band,

$$
<S>=\int_{B} S(v) d v=\int_{\tau}|E(t)|^{2} d t
$$

where $\langle S\rangle$ is the average flux density over the considered band, and $E(t)$ is the electric field, after appropriate bandpass filtering. The block diagram of a total power receiver is shown in fig. 1 A filter limits the band $B$ over which the measurement is performed. The signal power is computed using a nonlinear component with a square law behavior, a low-pass filter removes all radio frequency spectral components, and an integrator averages the power signal over the integration time $\tau$.

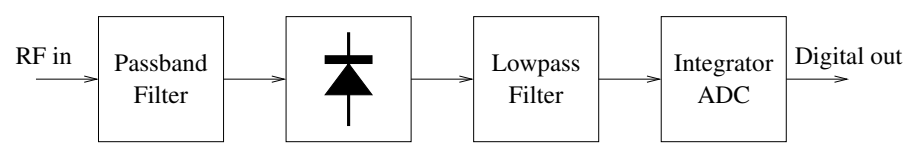

Figure 1: Total power detector block diagram 
The squaring device (detector) can be a diode, or a FET transistor, polarized in order to operate in a region where their response is approximately quadratic. The resulting circuit is simple, robust, and has a very wide intrinsic input bandwidth, up to tens of GHz. The quadratic law is however only approximate, and the accuracy may be degraded if the operating conditions deviate from the optimum point. For this reason, more complex analog multipliers have been adopted. These circuits are however more expensive, and have a maximum bandwidth of the order of a few $100 \mathrm{MHz}$.

The theoretical sensitivity of a detector with bandwidth $B$ and integration time $\tau$ is given by the radiometer equation:

$$
\Delta S=\frac{<S>}{\sqrt{B \tau}}
$$

Here $\langle S>$ includes (and is usually dominated) by the noise from the receiver, antenna and atmosphere. The equation is valid if the input noise spectrum is white, and if the system is stable in time. Especially the latter assumption is not true, as the receiver gain fluctuates in time with typically a $1 / f^{\alpha}$ spectrum, with $\alpha=0.5$ to 2 . For Indium Arsenide front-end amplifiers, these fluctuations have $\alpha \approx 1$, and translate in a gain noise of the order of a few milli-Kelvin of system temperature, with timescales ranging from hundreds of milliseconds to tens of seconds. For a bandwidth of $1 \mathrm{GHz}$ this noise overcomes the measurement error $\Delta S$ at an integration time of a second or even less. Additional source of $1 / f$ fluctuations are due to fluctuations in atmospheric transparency.

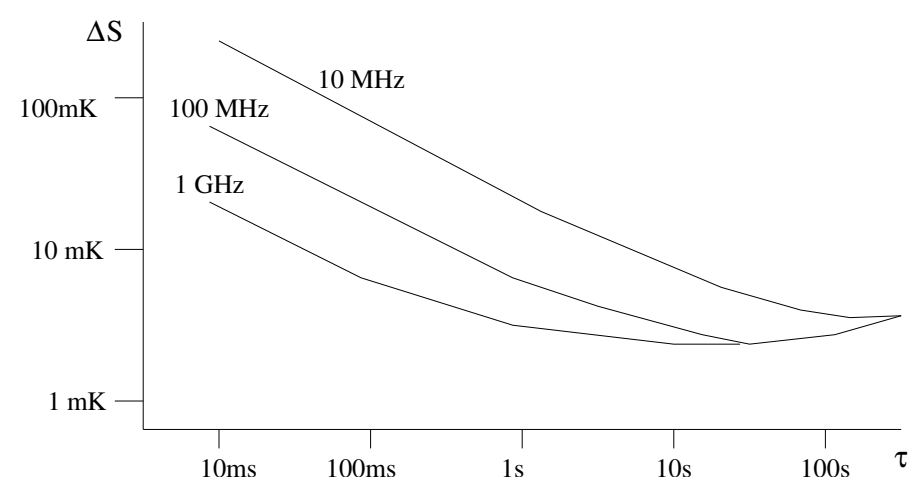

Figure 2: Typical total power sensitivity for a receiver with a system temperature of $20 \mathrm{~K}$, as a function of bandwidth and integration time. The sensitivity is limited by $1 / f$ noise for integration time longer than a few seconds

The problem exists for all observation techniques, but is particularly important when the band $B$ is larger. In spectroscopy the bandwidth in the radiometer equation refers to the equivalent width of a single spectroscopic point, $\Delta v$, typically in the $\mathrm{kHz}$ range, and the $1 / f$ noise becomes important only for integration time exceeding several minutes.

Most of the power measured by the total power detector is actually undesired noise: thermal emission from the atmosphere, from the ground entering the receiver through unwanted reflections and spillover, from the antenna itself, and especially noise contribution from the receiver. The actual astronomic signal is separated from all these contributions by subtracting the signal coming from a suitable nearby position on the sky. 
The $1 / f$ noise seriously degrades this subtraction, if its amplitude is larger than the radiometer noise. This limits the maximum integration time for the "on source" and "off source" observations. On the other side, the dead times involved in the position switch impose a minimum integration time. For fast calibration, a noise source is therefore added to the astronomic signal, on timescales up to a few milliseconds, but significantly increasing the total receiver noise.

For a more detailed analysis of the $1 / f$ noise and of the techniques adopted to remove it, see also the lecture by Natale[4] in this school.

\subsection{Polarimeter}

The receiver is sensitive to only one specific polarization of the incoming radio signal. Usually two receivers are employed, one for each of two orthogonally polarized components. For VLBI receivers, usually the two circular polarization components are used, while for millimetre receivers two linear polarizations are selected. The advantage of using circular polarizations is that the rotation of the antenna with respect to the sky produces only a slow phase change, and no derotation is needed to follow the change in parallactic angle as the antenna tracks the source. For circular polarization a polarizer (quarter-wave shifter) must be present in the antenna feed system, while for linear polarization the feed can be directly coupled to the receiver.

The polarization status is usually described by the 4 Stokes parameters: I, representing the total incoming power, $U$ and $Q$, describing the linear polarization and its direction, and $V$, describing the circular polarization (see for example [2]). These parameters are directly linked to the quantities:

$$
S_{i j}=\int E_{i}(t) E_{j}^{*}(t) d t
$$

where $E_{i j}$ are the electric fields in the two polarizations (e.g. $E_{L}, E_{R}$ for left and right circular polarizations). A good polarimetric receiver must provide very good equalization of the two receiver channels, amplitude and phase stability, and insulation of the two polarizations. These effects must be measured and removed by suitable calibration of polarized and unpolarized sources (see for example the excellent paper by Hamaker[3]).

Thus, to completely determine the polarization status one must compute the total power in the two polarization channels, and their complex cross correlation. An example of a polarimetric receiver is shown in fig. 2.2.

Maximum bandwidth of a polarimetric receiver depends on two factors: the bandwidth of analog multipliers and the Faraday rotation, that may produce an appreciable effect for bandwidths of tens to hundreds of $\mathrm{MHz}$. Therefore a continuum polarimetric receiver must provide a few separate bands, to measure and compensate this effect.

In general, any backend can be modified to measure the polarization parameters by including a cross correlation measurement of the two polarization channels, as in fig. 2.2. We have then polarimetric spectrometers, or polarimetric interferometers, that respectively measure the polarization properties of the frequency spectrum, or of the interferometric image.

\section{Spectroscopy}

A spectroscopic backend provides a measure of $S(v)$ as a function of the frequency $v$. It can be represented conceptually as an array of $N$ narrow band filters (spectral points), each with a 


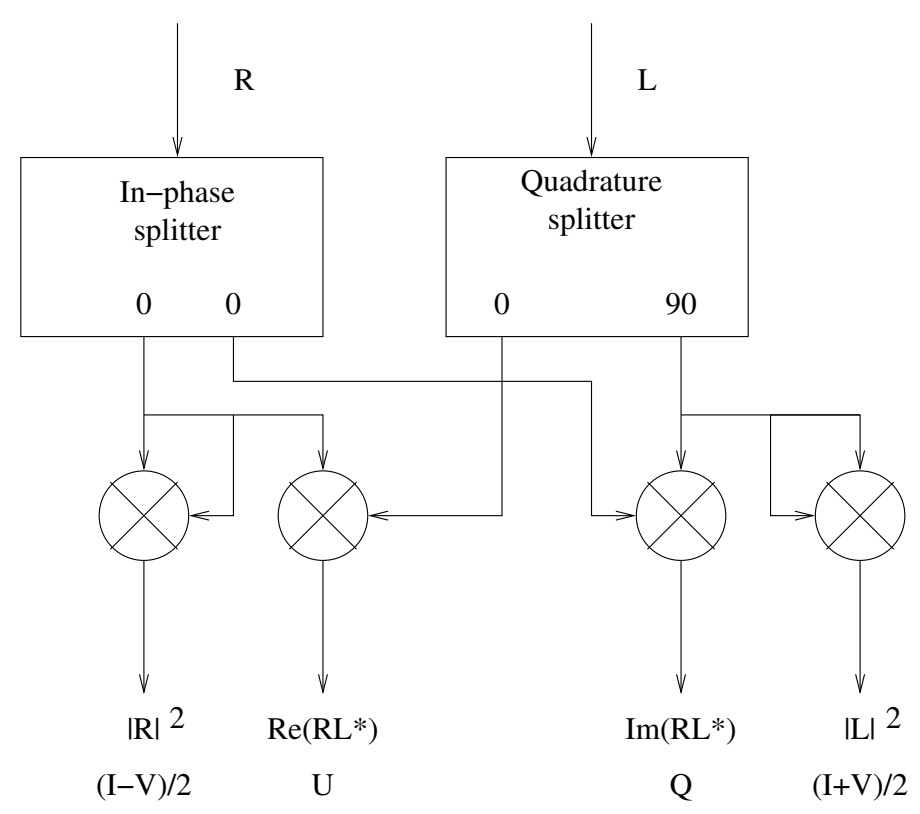

Figure 3: Polarimetric backend. Assuming input signals are circularly polarized, two total power detectors produce $(I \pm V) / 2$, while the complex correlation of the two signals produce the two linear polarization parameters $U$ and $Q$

resolution $\delta v$ and usually spaced on a regular grid with step $\Delta v=B / N$. The resolution is usually larger than the step, i.e. the spectral points are slightly overlapped.

There are two technological approaches to implement a radio spectrometer. For wide bandwidth ( $B$ of the order of $1-10 \mathrm{GHz}$ ) acousto-optic spectrometers are widely used, while for smaller bandwidths, and for interferometric applications, digital techniques are employed.

\subsection{Acousto-optic spectrometers}

In these instruments the radio signal is converted to an acoustic wave, travelling in a suitable crystal. The density modulations in the crystal act a $s$ a diffraction grating, deflecting a laser beam that is then focused on a CCD detector. The amplitude of the diffracted beam at each angle is proportional to $S(v)$, and the image on the CCD is then a direct representation of the power spectrum of the original radio wave.

The instrument, whose schematic is shown in figure 4, is compact, robust and conceptually very simple. The number of spectral points $N$ is however limited by the acoustic absorption of the crystal, and is generally of the order of 1000 . Band and number of channels are determined by the physical design, and cannot be changed for a given instrument.

It is possible to have several (2-4) waves travelling on a single crystal, thus increasing both $B$ and $N$. In figure 5 is shown the structure of the 4 channel AOS spectrometer for the Herschel HIFI instrument, with a total band $B=4 \mathrm{GHz}$ and $N=4096$.

\subsection{Digital spectrometers}

Digital spectrometers operate on a digitized representation of the radio signal. The main lim- 


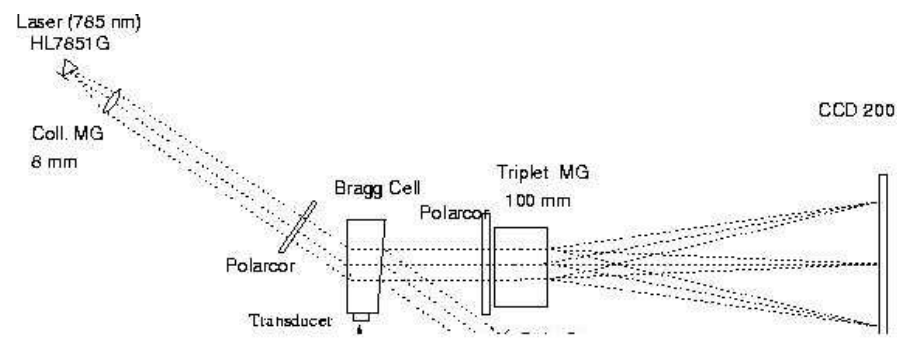

Figure 4: Concept of an acousto-optic spectrometer. A laser beam is diffracted by the acoustic replica of the radio wave. The diffracted pattern is focused on a CCD detector, where it forms the intensity spectrum of the radio signal

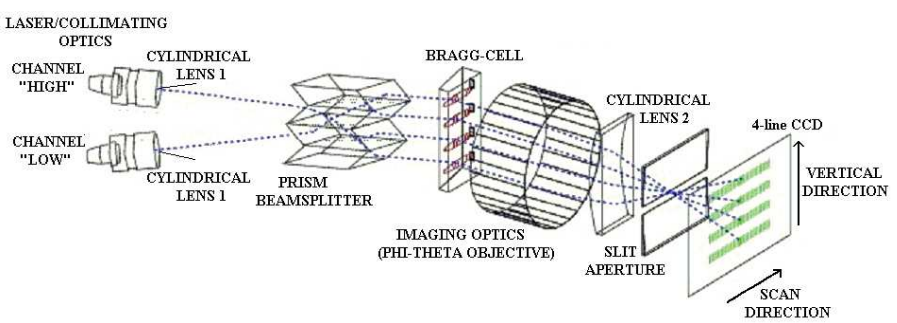

Figure 5: Acousto-optic spectrometer for the Herschel HIFI instrument

itation of these instruments is given by the clock frequency of the digital processing hardware that translates, for current available technology, to a bandwidth of a few $100 \mathrm{MHz}$. Higher bandwidths are possible using parallel processing techniques, but the complexity of the system increases rapidly with the parallelism required. For example the ALMA interferometer correlator analyzes a $8 \mathrm{GHz}$ bandwidth using 4 separate correlators, each composed by 32 parallel planes.

The main advantages of these instruments are the very high spectral resolution that can be achieved, now of the order of tens of thousands of spectral points, and their flexibility. The bandwidth and spectral resolution can be changed simply by changing the signal sampling frequency. A typical instrument is composed of many cascadable hardware modules, that can be combined in many possible ways in order to trade operational parameters, e.g. splitting the instrument into separate sections to analyze several independent spectral features with limited spectral resolution, increase the overall bandwidth or obtain very high resolution on a single feature.

The simplest digital spectrometer is represented by an autocorrelator (fig. 6). The signal to be analyzed is delayed, using a digital delay line, and the product of each delayed output is multiplied with the original undelayed signal. The instrument thus computes autocorrelation function

$$
A(\tau)=\int_{T} E(t) E(t-\tau) d t
$$

on a discrete number of delays $\tau=n \Delta \tau$ whose Fourier transform is the power spectral density $S(v)$. As the operations performed on the instrument are a multiplication followed by a Fourier transform, this approach is usually denoted as XF correlation. The bandwidth of the instrument is $1 / \Delta \tau$, and the number of spectral points is equal to the number of delays computed, $N=n_{\max }$. 


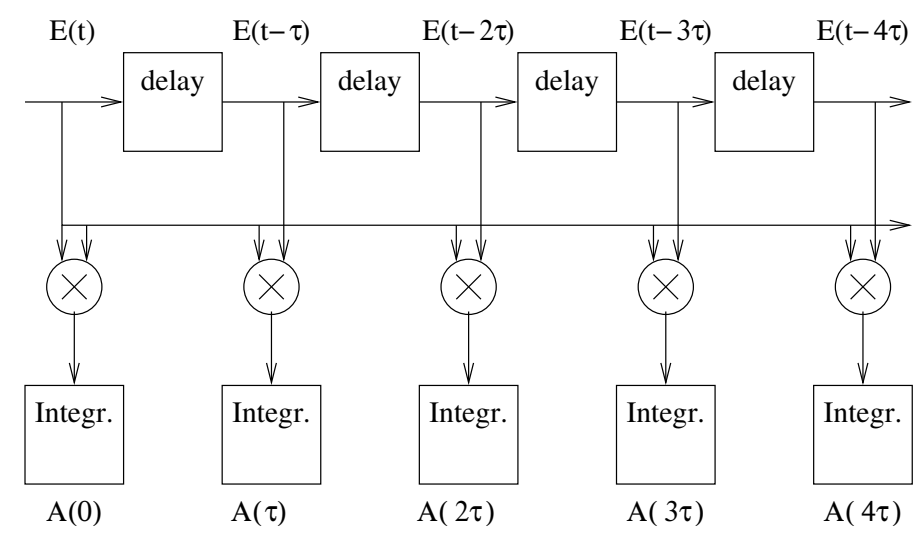

Figure 6: Autocorrelation spectrometer. The signal is delayed in a series of discrete delay blocks, and multiplied by the original undelayed signal

For a radio signal that is basically a Gaussian noise, it is sufficient to represent the digital samples with 1 or 2 bits. The hardware required for the delay line and the multipliers is thus very simple, allowing high operational speeds and a large number of spectral points $N=10^{3}-10^{4}$. The rough quantization causes an increase in the overall noise, respectively of $57 \%$ and $21 \%$ for 1 and 2 bit quantization.

With the advent of fast digital multipliers, it has been possible to build a device that computes the Fourier transform on a radio frequency signal in real time. This Fourier engine accepts $N$ subsequent samples of the signal and produces a $N$ point amplitude spectrum of the same (fig. 7). The spectrum is then squared and accumulated in an integrator. As the order of the Fourier transform and of the multiplication is inverted with respect to the autocorrelation spectrometer, this approach is denoted as FX correlation.

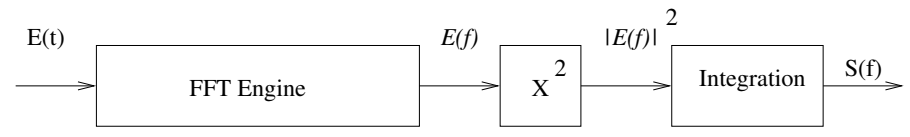

Figure 7: Fourier transform spectrometer. A Fourier engine computes the instantaneous amplitude spectrum of the incoming radio data, that is subsequently squared and integrated

The Fourier spectrometer has several advantages over a correlator. The hardware complexity is proportional to $\log _{2}(N)$, so even if the single FFT stage is much more complex than a single correlator stage, it is easier to build instruments with very high spectral resolution, up to millions of spectral points. There is no advantage of using representations with a small number of bits, so the dynamic range of the instrument is much larger, with very good properties of immunity to interferences.

The FFT processor operates on a finite segment of the input signal. The resulting spectral response of a single point has naturally a $\sin (x) / x$ shape, and a strong monochromatic signal can contaminate the whole spectrum. Therefore in recent instruments the FFT processor is preceded by a small filter, in the so called polyphase design. This is equivalent to applying a long convolutional 
window to the data, and makes possible to control the individual spectral point response. In this way the single point has an almost rectangular response, with very high rejection of signals from adjacent points and an immunity to unwanted signals (RFI) of up to $10^{9}$

Both XF and FX spectrometers can be used to compute the cross spectrum of two signals, i.e. to compute the cross polarization terms in a polarimeter. In a XF spectrometer, the two signals are applied respectively to the undelayed branch and to the digital delay line, while in a FX spectrometer two separate FFT engines are used to compute the two spectra, that are subsequently multiplied together.

\section{Interferometry}

In an interferometer two or more antennas are connected together, in order to achieve larger spatial resolution. It can be shown that the cross spectrum $C_{i j}$ of the signals received by two antennas $i$ and $j$ :

$$
C_{i j}(v)=\int E_{i}(v) E_{j}^{*}(v) d v
$$

is, in the approximation of a flat sky, the Fourier transform component of the flux density distribution computed at the angular frequency $\left(X_{i}-X_{j}\right) / \lambda$, where $X(i)$ and $X(j)$ are the spatial coordinates of the two antennas, $\lambda$ the observing frequency wavelength, and the reference point of the map is perpendicular to $\left(X_{i}-X_{j}\right)$.

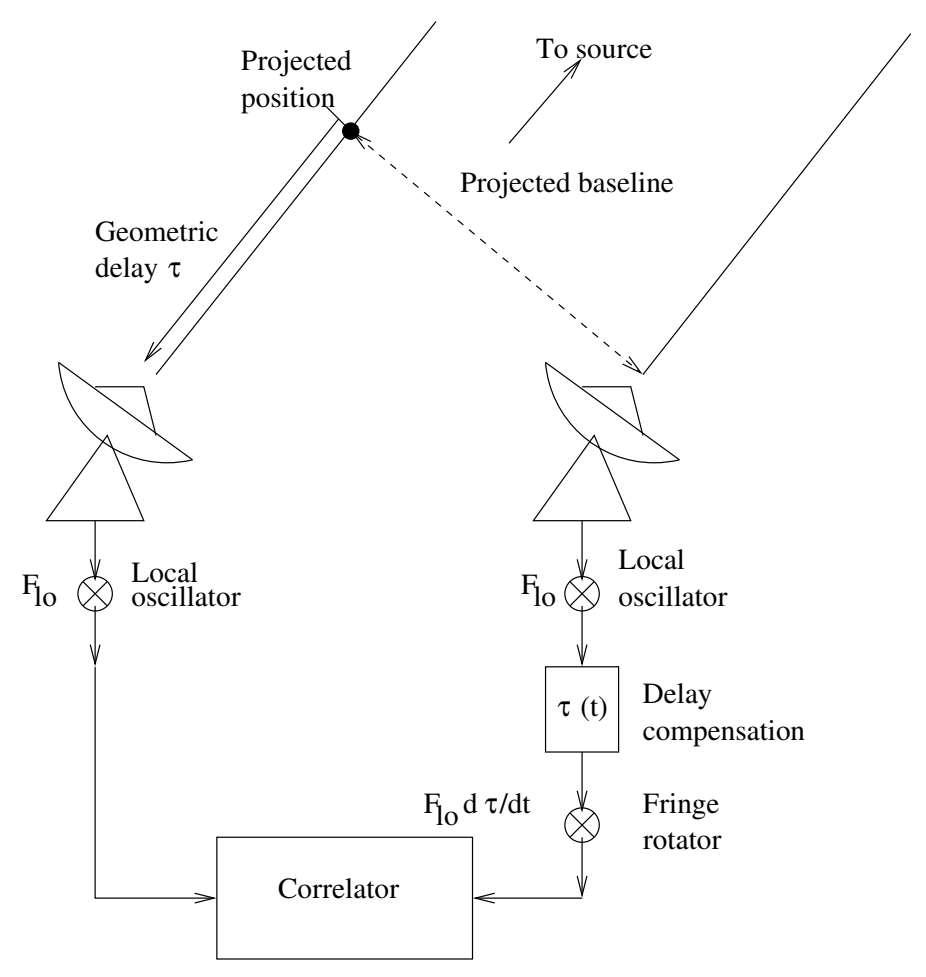

Figure 8: Interferometer conceptual design. Two antennas observe the same source, and the received signal is correlated (multiplied) after compensating the geometric delay $\tau$. The fringe rotator corrects the phase of the local oscillator, seen through a time varying delay 
In a practical interferometer there are several complications. The region of interest is not usually perpendicular to the vector joining the two antennas, One (or both) are thus moved electronically along the line of sight, inserting an appropriate delay in the data path. As the Earth rotates, the delay must be continuously changed to track the source.

The delay is usually applied after the frequency conversion in the receiver. The phase of the receiver local oscillator, seen through a varying delay, changes with time and must be compensated for in a fringe rotator, a separate local oscillator with a frequency

$$
v_{f}=v_{l o} d \tau / d t
$$

The complete conceptual schematic for a two antenna correlator is shown in fig. 8. Typically a modern correlator is composed of 4-80 antennas, interconnected to form all $n(n-1) / 2$ possible baselines. As the Earth rotates, the correlator orientation changes, and different components of the image transform are observed. Interferometric images can reach very high image fidelity, with dynamic ranges in excess of $10^{5}$ (see for example [5]).

The correlation process can be physically performed in two ways: using a cross correlator, analogue to the autocorrelator described in chapter 3.2 (XF correlator), or computing the Fourier transform of the two radio signals and multiplying them directly for each frequency bin (FX correlator). FX correlators need a more complex electronics, that is replicated for each antenna, while very simple electronics is required for each baseline. XF correlators have simpler electronics, but this must be replicated for each baseline. As baseline number increases with the square of the antennas, FX architecture is favored for correlators with a large number of antennas. FX architecture allows a better control of several instrumental effects that depend on the frequency, and a much higher spectral resolution.

\subsection{Patch antenna arrays}

Using an array of very small, omnidirectional antennas it is possible to synthesize a reasonable aperture by summing all the signal together. The signal from each antenna is processed as in fig. 8 , and the sum is performed weighting each signal in a controlled way. In this way the telescope can be steered electronically over a large part of the sky, without any moving part.

This architecture has several advantages:

- As no mechanical parts are involved, the antenna pattern can be steered very quickly, allowing for example very fast on-off source cycles.

- More than a single beam can be synthesized, allowing several, separate, regions of the sky to be observed simultaneously. This is useful, for example, for phase referencing of VLBI observations.

- It is possible to control the beam shape in order to place nulls of beam sensitivity in the direction of interfering sources (e.g. commercial radio stations).

This architecture is generally used for elements of large interferometers (LOFAR, SKA), as the absence of moving parts increases antenna reliability and security. It is however difficult to implement at frequencies higher than a few $\mathrm{GHz}$, and for large bandwidths. 


\section{Digital vs. Analog}

Digital signal processing is already used in several of the instruments described above. Digital spectrometers and interferometric correlators are all based on the numeric processing of digital samples.

In recent years, due to the availability of cheap and large digital programmable components, it has become possible to perform all the data processing and analysis described above using digital signal processing. In particular Field Programmable Gate Arrays (FPGA), that are generic programmable chips containing tens to thousands of individual multipliers, plus generic logic that can be arranged as adders, comparators, integrators, etcetera, are extensively used. It is thus possible to implement a generic algorithm directly in hardware, and to replace analog components, like filters, mixers, local oscillators, with their numeric counterparts.

Pushing this tendency to its extreme, a complete radio receiver can be built using only a direct amplifier (without frequency conversions), a sampler, and a lot of digital processing hardware. This is possible today for sky frequencies of up to several $\mathrm{GHz}$, and for instantaneous bandwidths of 1-2 $\mathrm{GHz}$, but these limits are quickly increasing. Such an instrument is called a digital receiver, and has several advantages over its analog counterpart.

- The performances that can be obtained with a digital filter are usually much better, in terms of bandpass flatness, stop-band rejection, steepness of the transition region, than those of an analog component

- As the processing is fully deterministic, and stable with time, the instrument response can be exactly calculated and removed with good accuracy without the need of a calibration measurement.

- A digital system is generally more immune to nonlinear effects than its analog counterpart. This increases its immunity to strong interfering signals. On the other hand, when nonlinear effects are present, they are more severe and difficult to correct in a digital system.

- Digital systems interface easily with the computer system that processes the data. Data processing can be easily moved from hardware to software and back depending on the speed and complexity requirements.

A digital system implemented using programmable devices can be described using high level hardware description languages, or HDL. A HDL program describes the processing performed on the radio signal in an abstract, mathematical way, and can be translated to hardware configuration by suitable compilers. The same hardware can be used to implement completely different instruments, like a spectrometer, a correlator, or a polarimeter, simply by changing the program. Different hardware components can be collected in a public library and combined together in a more complex entity like the subroutines of a program. This is analogue to what happened when programmable computers replaced mechanical computing machines.

Several general purpose platforms are being developed. Among them, the Berkeley BEE machine[1], a development board containing 5 large programmable chips, can implement a rich set of instruments based on a public library. The instrument behavior is described at high level 
in mathematical form using a general purpose language like MatLab, and this is translated by a specific software to the required instrument.

A smaller example is the Digital BBC system, developed by the European VLBI Network and initially conceived as a all-digital replacement for a VLBI terminal. The system, including a fast sampler and individual modules containing a single FPGA, can be used as a general-purpose, modular back-end.

\section{References}

[1] Chen Chang, John Wawrzynek, and Robert W. Brodersen, 2005, "BEE2: A High-End Reconfigurable Computing System", IEEE Design \& Test, 22/2, 114

[2] Kraus, J. D., Radio Astronomy, McGraw Hill Book Company, 1966

[3] Hamaker, J. P., Bregman, J. D. Sault, R. J. 1996, A\&ASS, 117, 137

[4] Natale, E., 2007 this school

[5] Perley, R. A., 1999, "High Dynamic Range Imaging", in "Synthesis Imaging in Radio Astronomy II", ASP Conf. Ser. 180, 275

[6] Romney, J. D., 1999, "Cross correlators", in "Synthesis Imaging in Radio Astronomy II", ASP Conf. Ser. 180, 57 